\title{
Evaluation of $\mathrm{Gd}_{2} \mathrm{SiO}_{5}: \mathrm{Ce}(\mathrm{GSO}), \mathrm{Lu}_{2-x} \mathrm{Gd}_{x} \mathrm{SiO}_{5}: \mathrm{Ce}$ (LGSO), and $\mathrm{Lu}_{2-x} \mathrm{Y}_{x} \mathrm{SiO}_{5}: \mathrm{Ce}$ (LYSO) Crystalline Scintillators Using a Photoacoustic Spectroscopy Technique
}

\author{
Yutaka Fujimoto, ${ }^{1 *}$ Daisuke Nakauchi, ${ }^{2}$ Takayuki Yanagida, ${ }^{2}$ \\ Masanori Koshimizu, ${ }^{1}$ and Keisuke Asai ${ }^{1}$ \\ ${ }^{1}$ Department of Applied Chemistry, Graduate School of Engineering, Tohoku University, Sendai 980-8579, Japan \\ ${ }^{2}$ Graduate School of Materials Science, Nara Institute of Science and Technology, \\ 8916-5 Takayama, Ikoma, Nara 630-0192, Japan
}

(Received January 31, 2021; accepted May 25, 2021)

Keywords: photoacoustic spectroscopy, thermal deactivation, scintillator

We evaluated the nonradiative deactivation in three scintillators, $\mathrm{Gd}_{2} \mathrm{SiO}_{5}: \mathrm{Ce}$ (GSO), $\mathrm{Lu}_{2-x} \mathrm{Gd}_{x} \mathrm{SiO}_{5}: \mathrm{Ce}$ (LGSO), and $\mathrm{Lu}_{2-x} \mathrm{Y}_{x} \mathrm{SiO}_{5}$ :Ce (LYSO), using a photoacoustic spectroscopy technique (PAS). The photoacoustic (PA) spectra suggested that part of the absorption energy for the excitation of $\mathrm{Ce}^{3+}$ will be released as heat through a nonradiative deactivation process. GSO exhibits an intense PA signal compared with LGSO and LYSO because of its energy migration process from $\mathrm{Gd}^{3+}$ to $\mathrm{Ce}^{3+}$ emission centers.

\section{Introduction}

Scintillators are materials with the capability to convert energy from ionizing radiation such as X-rays and gamma rays into pulses of light commonly known as ultraviolet-visible photons. The scintillation process in the materials usually consists of three complex physical steps. ${ }^{(1)}$ The initial step is energy deposition in the scintillation material due to the interaction between the incident ionizing radiation and the material. As a result of the energy deposition, many excited electrons are generated in the scintillation material. The electron-hole pair is also referred to as an exciton. The next step is thermal relaxation and energy transfer of the excited electrons. The electrons interact with the host lattice and other electrons, resulting in the creation of secondary electrons or phonons. The final step is recombination and luminescence. The luminescence is mainly generated by two processes: one is the recombination of electrons and holes; the other is the excitation of luminescent centers in the scintillation material by interactions with the secondary electrons. Although the fundamental phenomenon of scintillation is understood, there are no theories to predict the energy conversion efficiency of scintillators. Robbins ${ }^{(2)}$ and Lempicki and coworkers ${ }^{(3,4)}$ derived a phenomenological model of scintillation efficiency, which is formulated as $L Y_{S C}=\beta \times S \times Q$, where $L Y_{S C}$ is the scintillation light yield, $\beta$ is the efficiency of the conversion process, $S$ is the energy migration efficiency from the host to the luminescent

*Corresponding author: e-mail: fuji-you@qpc.che.tohoku.ac.jp https://doi.org/10.18494/SAM.2021.3321 
centers, and $Q$ is the fluorescence quantum efficiency $(\mathrm{QE})$ of the luminescent centers. The conversion efficiency $\beta$ can be expressed by $\beta=n_{e-h} /\left(E / 2.3 E_{g}\right)$, where $n_{e-h}$ is the number of electron-hole pairs created upon exposure to ionizing radiation, $E$ is the energy deposited by the ionizing radiation, and $E_{g}$ is the band-gap energy of the material. The phenomenological model has been used to explain the light yield (LY) of developed scintillators and for the design of novel materials by many researchers. However, an ideal scintillator that can the fulfill the requirements for ionizing radiation detection in various applications does not yet exist. Therefore, there is still a need to study the physical characteristics of scintillators via a lateral approach. We have recently focused on a photoacoustic spectroscopy (PAS) technique ${ }^{(5,6)}$ that can directly measure the energy loss and nonradiative deactivation of the luminescent centers in samples. The photoacoustic (PA) effect refers to the generation of heat through the interaction of light with matter. This heat generates a pressure wave or sound in the surrounding gaseous layer, which can be detected with an electret microphone as a PA signal. The first observation of the PA effect was in the eighteenth century when Bell found that sound could be induced in a sample by illumination with modulated sunlight. ${ }^{(7)}$ A similar effect was confirmed in other forms such as gases and liquids by many researchers. ${ }^{(8)}$ In 1976, Rosencwaig and Gersho derived their thermal piston model for the one-dimensional heat flow analysis of a PA signal in a cylindrical solid-gas interface. ${ }^{(9)}$ PAS has proved to be a powerful technique for measuring optical absorption spectra and directly observing the nonradiative deactivation process in semiconductor and phosphor materials. ${ }^{(10-18)}$ In this study, we evaluated three scintillators, $\mathrm{Gd}_{2} \mathrm{SiO}_{5}$ : $\mathrm{Ce}$ (GSO), $\mathrm{Lu}_{2-x} \mathrm{Gd}_{x} \mathrm{SiO}_{5}$ :Ce (LGSO), and $\mathrm{Lu}_{2-x} \mathrm{Y}_{x} \mathrm{SiO}_{5}$ :Ce (LYSO), using a PAS detection system and attempted to clarify the origin of nonradiative deactivation. Finally, the relationship between the PA signal intensity, the fluorescence QE value, and the scintillation LY is discussed.

\section{Methods}

The scintillator samples of GSO and LGSO were prepared by OXIDE Corporation by the conventional Czochralski growth technique. The sample of the GSO was a commercialized product for gamma-ray spectroscopy measurement, whereas the LGSO sample was a trial product with a slightly different chemical composition from that of the product commercialized by OXIDE Corp. for the present positron emission tomography (PET) scanner. A LYSO commercial scintillator was used for comparison in all measurements. The PA spectra of the samples were measured using our original setup. ${ }^{(6)} \mathrm{A} 300 \mathrm{~W}$ xenon arc lamp with an aspherical condensing mirror equipped with a monochromator (SM-5 High-Power Monochromator, Bunkoukeiki) was used as the excitation light source. The monochromatic light passed through a mechanical chopper (BCH-VL, Bunkoukeiki) and an optical fiber, producing a modulating light that was focused onto a sample inside a laboratory-built airtight PA cell through a quartz window. The PA signal from the sample was detected with an electret microphone (UC-59, Rion) connected to a preamplifier (NH-22A, Rion) and a sound level meter unit (UN-14, Rion), and processed by a two-phase lock-in amplifier $(5610 \mathrm{~B}, \mathrm{NF})$ with a reference signal input from the chopper. The PA signal was recorded at $19 \mathrm{~Hz}$ at a scanning rate of $2 \mathrm{~nm} / \mathrm{min}$. The PA signal intensity is usually proportional to the excitation light intensity and the quantity of heat 
generated due to the thermal deactivation process of the sample; hence, it is also proportional to the optical absorption of the sample. Thus, all spectra were corrected for the wavelength dependence of both the excitation light intensity using a carbon black standard and the diffuse reflectance of the sample. For the correction of the PA spectrum data, the diffuse reflectance spectra of the samples were measured with a UV-Vis spectrophotometer (UV-2700, Shimadzu) equipped with an integrating sphere unit (ISR-2600, Shimadzu). $\mathrm{BaSO}_{4}$ was used as the reference standard. The excitation spectra of the samples were obtained with a fluorescence spectrophotometer (F-7000, Hitachi). The fluorescence QE of the samples was determined with a spectrofluorometer (Quantaurus-QY, Hamamatsu) equipped with a calibrated integrating sphere. The scintillation LY of the samples was estimated by measurements of the ${ }^{137} \mathrm{Cs}$-gammaray-induced scintillation pulse height spectrum, as explained in Refs. 19 and 20. A metal package photomultiplier tube (R7600U-20, Hamamatsu) was used as a photosensitive detector, which was operated at $600 \mathrm{~V}$. The output signal was amplified and shaped with a shaping time of $2 \mu$ s by a preamplifier (ORTEC 113) and a shaping amplifier (ORTEC 572). Finally, pulse height spectra were obtained with a multichannel amplifier (MCA8000D, Amptec). In the measurement, a NaI:Tl commercial scintillator was used as a standard sample to calculate the scintillation LY.

\section{Results and Discussion}

Figure 1(a) shows the PA spectra of the GSO scintillator, with the excitation spectra also presented for comparison. The PA bands were observed in the 235-250, 275-290, and 320-360 $\mathrm{nm}$ wavelength regions, which are roughly consistent with the excitation bands appearing at 220-265, 270-300, and 325-355 nm, respectively. Many researchers have studied the excitation process and energy migration mechanism in GSO by performing optical absorption and photoluminescence measurements at low temperatures. ${ }^{(21-26)}$ Firstly, the excitation band at $325-355 \mathrm{~nm}$ originates from electron transition from the $4 \mathrm{f}\left({ }^{2} \mathrm{~F}_{5 / 2}\right)$ ground state to the $5 \mathrm{~d}^{1}$ excited state of $\mathrm{Ce}^{3+}$. Secondly, the excitation band at $270-300 \mathrm{~nm}$ corresponds to transitions from the ${ }^{8} \mathrm{~S}_{7 / 2}$ ground state to the ${ }^{6} \mathrm{I}_{\mathrm{j}}$ multiples of $\mathrm{Gd}^{3+}$, which overlap the absorption band of the $5 \mathrm{~d}^{2}$ excited state of $\mathrm{Ce}^{3+}$, resulting in nonradiative energy transfer from $\mathrm{Gd}^{3+}$ to $\mathrm{Ce}^{3+}$ emission centers. Finally, the excitation band at $220-265 \mathrm{~nm}$ is due to the overlap between the absorption band of the $5 \mathrm{~d}^{3}$ excited state of $\mathrm{Ce}^{3+}$ and the electron transitions from the ${ }^{8} \mathrm{~S}_{7 / 2}$ ground state to the ${ }^{6} \mathrm{D}_{\mathrm{j}}$ multiples of $\mathrm{Gd}^{3+}$. This result suggests that part of the absorption energy for the excitation will be released as heat through the nonradiative transition and Stokes shift of the $\mathrm{Ce}^{3+}$ emission and nonradiative deactivation during the energy migration from $\mathrm{Gd}^{3+}$ to $\mathrm{Ce}^{3+}$ emission centers. In particular, because of the intense PA bands at 220-265 and 270-300 nm, GSO was found to lose a large amount of energy due to the energy migration between $\mathrm{Gd}^{3+}$ and $\mathrm{Ce}^{3+}$. Similarly, the PA and excitation spectra of the LGSO and LYSO scintillators are shown in Figs. 1(b) and 1(c), respectively. The PA spectra exhibited three bands at 250-275, 280-320, and $340-375 \mathrm{~nm}$, whereas a prominent excitation band appeared at 330-380 nm. Moreover, at least two weak excitation bands can be observed at 250-275 and 280-320 $\mathrm{nm}$. These excitation bands are very similar to those of the $\mathrm{Lu}_{2} \mathrm{SiO}_{5}$ :Ce scintillator measured by Suzuki et al. and Melcher et al. ${ }^{(27,28)}$ They correspond to the electron transition from the $4 \mathrm{f}\left({ }^{2} \mathrm{~F}_{5 / 2}\right)$ ground state to the $5 \mathrm{~d}^{1}$ 


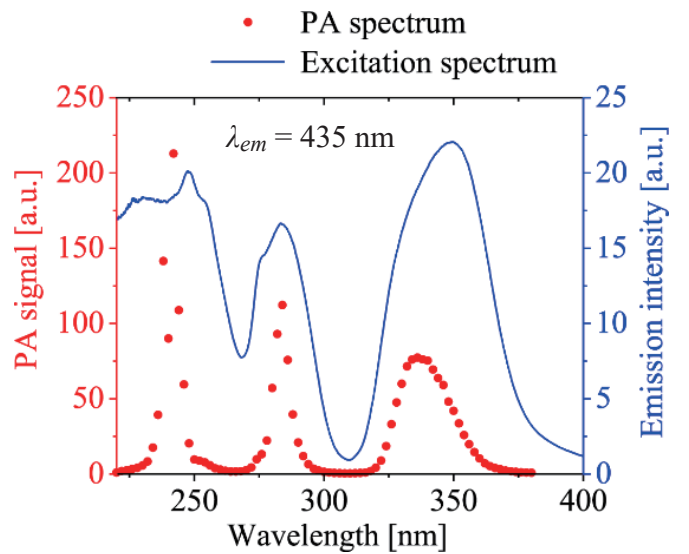

(a)

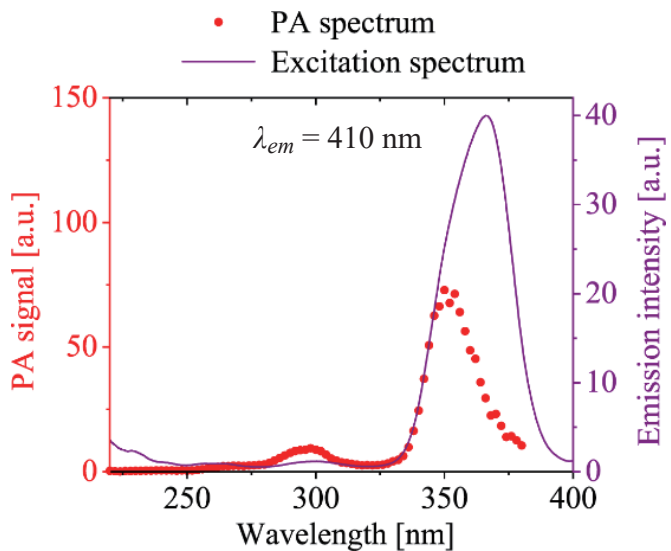

(b)

- PA spectrum

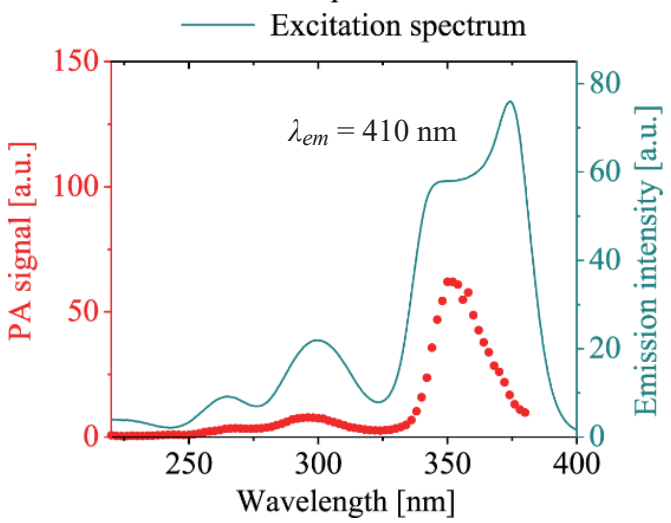

(c)

Fig. 1. (Color online) PA and excitation spectra of (a) GSO, (b) LGSO, and (c) LYSO scintillators.

(330-380 nm), 5d $\mathrm{d}^{2}(280-320 \mathrm{~nm})$, and $5 \mathrm{~d}^{3}(250-275 \mathrm{~nm})$ excited states of $\mathrm{Ce}^{3+}$. The PA bands overlapped with the $\mathrm{Ce}^{3+}$ excitation bands, indicating that the heat generated in LGSO and LYSO is mainly caused by the nonradiative transition and Stokes shift of the $\mathrm{Ce}^{3+}$ emission centers. Unlike the GSO scintillator, LGSO showed no intense PA signals at the excitation bands of $5 \mathrm{~d}^{2}$ and $5 \mathrm{~d}^{3}$ in $\mathrm{Ce}^{3+}$. This is because the energy migration from $\mathrm{Gd}^{3+}$ to $\mathrm{Ce}^{3+}$ emission centers should be weak due to the low $\mathrm{Gd}^{3+}$ concentration in the LGSO host.

Figure 2 shows the relationship between the PA signal intensity and fluorescence QE value of the $\mathrm{Ce}^{3+}$ emission due to electron transitions from the $5 \mathrm{~d}^{1}$ lowest excited state to the ${ }^{2} \mathrm{~F}_{5 / 2}$ and ${ }^{2} \mathrm{~F}_{7 / 2}$ 4f ground states in the GSO, LGSO, and LYSO scintillators. In the figure, the $x$ and $y$ axes indicate the PA signal intensity and QE value, respectively. The excitation wavelengths in the measurements were $336 \mathrm{~nm}$ for GSO and $352 \mathrm{~nm}$ for LGSO and LYSO. The fluorescence QE values were calculated as the ratio of the number of photons emitted from the sample to the number of photons absorbed by the sample. From the calculation, the fluorescence QE values of GSO, LGSO, and LYSO were 82.8, 88.6, and 90.6\%, respectively. In contrast, the PA signal intensity of the samples shows an inverse correlation with the QE value, which is reasonable considering the energy conservation of the excitation energy. We previously confirmed similar results for $\mathrm{Cs}_{2} \mathrm{HfCl}_{6}$ :Te and $\mathrm{Y}_{3} \mathrm{Al}_{5} \mathrm{O}_{12}$ :Ce. ${ }^{(5,6)}$ 


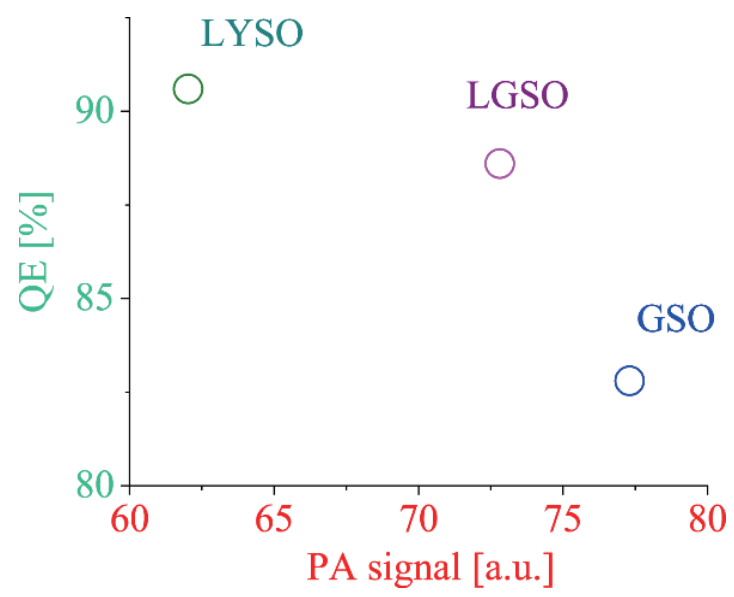

Fig. 2. (Color online) Relationship between PA signal intensity and fluorescence QE value of $\mathrm{Ce}^{3+}$ emission in GSO, LGSO, and LYSO scintillators.

Figure 3 shows the values of the integrated PA signal intensity in the $220-380 \mathrm{~nm}$ wavelength ranges of the GSO, LGSO, and LYSO scintillators. The intensity of the PA signal of the GSO scintillator was more than twice that of the LGSO and LYSO scintillators. A possible explanation for this is that the integrated values include not only the PA signal due to the nonradiative transition and Stokes shift of the $\mathrm{Ce}^{3+}$ emission but also that of other nonradiative deactivation processes. As described above, GSO exhibits intense PA bands at 220-265 and 270-300 nm, originating from the energy loss during the energy migration from $\mathrm{Gd}^{3+}$ to $\mathrm{Ce}^{3+}$. For this reason, the total quantity of nonradiative deactivation of GSO might be larger than those of LGSO and LYSO. The relationship between the value of the integrated PA signal intensity and the estimated scintillation LY of the GSO, LGSO, and LYSO scintillators is shown in Fig. 4. From calculations and measurements of the pulse height spectra, the scintillation LYs of GSO, LGSO, and LYSO were found to be 8000,24300 , and 27800 photons/MeV, respectively, i.e., the scintillation LY of the scintillators was high when the PA signal intensity was low, indicating an inverse correlation. As described above, in earlier studies of the fundamental limit on the scintillation LY of scintillators, the following useful model was derived by Robbins and Lempicki and coworkers: ${ }^{(2-4)}$

$$
L Y_{S C}=E /\left(\beta E_{g}\right) \times S \times Q
$$

where $L Y_{S C}$ is the scintillation LY, $E$ is the energy deposited by ionizing radiation, $\beta$ is a constant parameter, $E_{g}$ is the band-gap energy of the scintillator, $S$ is the energy migration efficiency from the host to emission centers, and $Q$ is the fluorescence $\mathrm{QE}$ of the emission centers. From the model, the scintillation LY was found to be proportional to the energy migration efficiency from the host to the emission centers and the fluorescence QE of the emission centers. This suggests that the integrated PA signal intensity varies in inverse proportion to the estimated scintillation LY because the PA signal is due to the energy loss in both energy migration from the host to the emission centers and electron transition of the emission centers. Therefore, the relationship 


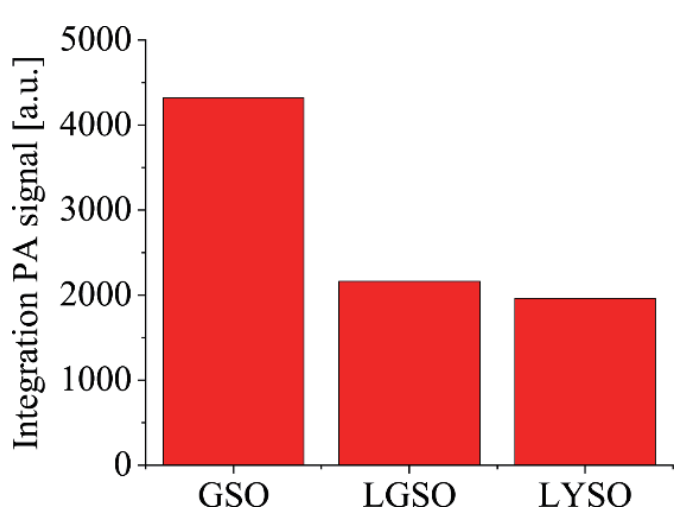

Fig. 3. (Color online) Comparison of integrated PA signal intensity in 220-380 $\mathrm{nm}$ wavelength range of GSO, LGSO, and LYSO scintillators.

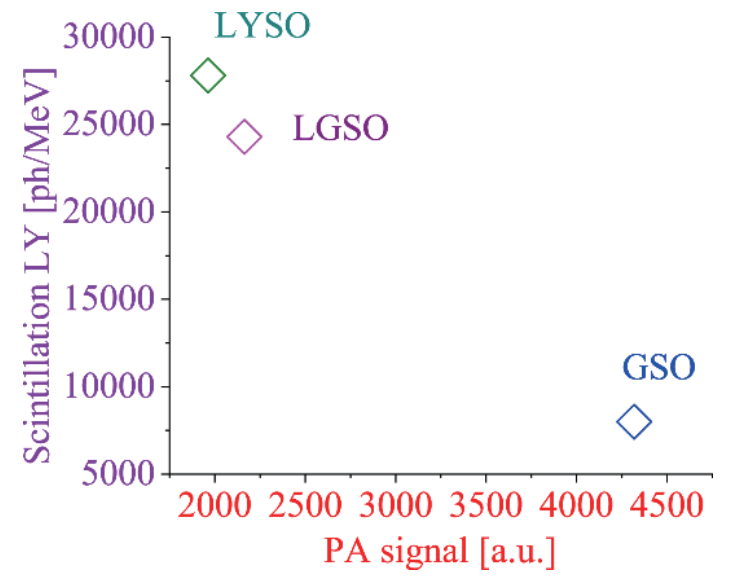

Fig. 4. (Color online) Relationship between integrated PA signal intensity and estimated scintillation LY of GSO, LGSO, and LYSO scintillators.

between the integrated PA signal intensity and the scintillation LY from GSO, LGSO, and LYSO observed in this study is consistent with the phenomenological model, which is a reasonable result based on energy conservation.

\section{Summary}

We detected the PA signals from the nonradiative deactivation process in GSO, LGSO, and LYSO commercial scintillators using a PAS system. From the PA and excitation spectra, the main origin of the nonradiative deactivation in LGSO and LYSO was found to be the nonradiative transition and Stokes shift of the $\mathrm{Ce}^{3+}$ emission. GSO showed intense PA signals originating from the energy migration between $\mathrm{Gd}^{3+}$ and $\mathrm{Ce}^{3+}$ in addition to the nonradiative deactivation of the $\mathrm{Ce}^{3+}$ emission. From the evaluation using GSO, LGSO, and LYSO, we experimentally confirmed that the PA signal intensity is strongly inversely correlated with the fluorescence QE and scintillation LY.

\section{Acknowledgments}

This work was supported by the Cooperative Research Project of the Research Center for Biomedical Engineering, Nakatani Foundation for Advancement of Measuring Technologies in Biomedical Engineering, and Terumo Life Science Foundation.

\section{References}

1. T. Yanagida: Proc. Jpn. Acad., Ser. B 94 (2018) 75.

2 D. J. Robbins: J. Electrochem. Soc. 127 (1980) 2694.

3 A. Lempicki, A. J. Wojtowicz, and E. Berman: Nucl. Instrum. Methods Phys. Res., Sect. A 333 (1993) 304.

4 A. Lempicki and A. J. Wojtowicz: J. Lumin. 60-61 (1994) 942.

5 Y. Fujimoto, K. Saeki, D. Nakauchi, H. Fukada, T. Yanagida, H. Kawamoto, M. Koshimizu, and K. Asai: Mater. Res. Bull. 105 (2018) 291. 
6 Y. Fujimoto, D. Nakauchi, T. Yanagida, M. Koshimizu, H. Fukada, Y. Hayashi, and K. Asai: Sens. Mater. 32 (2020) 1453.

7 A. G. Bell: Am. J. Sci. 20 (1880) 305.

8 M. L. Viengreov: Doklady Akademii Nauk SSSR 19 (1938) 687.

9 A. Rosencwaig and A. Gersho: J. Appl. Phys. 47 (1976) 64.

10 L. D. Merkle and R. C. Powell: Chem. Phys. Lett. 46 (1977) 303.

11 M. Morita: Jpn. J. Appl. Phys. 20 (1981) 296.

12 H. Tokumoto, M. Tokumoto, and T. Ishigura: Jpn. J. Appl. Phys. 50 (1981) 602.

13 O. Zelaya-Angel, J. J. Alvarado-Gil, R. Lozada-Morales, H. Vargas, and A. Ferreira da Silva: Appl. Phys. Lett. 64 (1994) 291.

14 A. Ferreira da Silva, N. Veissid, C. Y. An, I. Pepe, N. Barros de Oliveira, and A. V. Batista da Silva: Appl. Phys. Lett. 69 (1996) 1930.

15 F. Firszt, S. Łęgowski, H. Męczyńska, B. Sekulska, J. Szatkowski, J. Zakrzewski, and W. Paszkowicz: Acta Phys. Pol. A 92 (1997) 749.

16 J. J. Prías-Barragán, L. Tirado-Mejía, H. Ariza-Calderón, L. Baños, J. J. Perez-Bueno, and M. E. Rodríguez: J. Cryst. Growth 286 (2006) 279.

17 Z. J. Chen, J. W. Fang, and S. Y. Zhang: Int. J. Thermophys. 36 (2015) 947.

18 S. J. Zelewski and R. Kudrawiec: Sci. Rep. 7 (2017) 15365.

19 Y. Fujimoto, K. Saeki, D. Nakauchi, T. Yanagida, M. Koshimizu, and K. Asai: Sens. Mater. 30 (2018) 1577.

20 Y. Fujimoto, K. Saeki, D. Nakauchi, T. Yanagida, M. Koshimizu, and K. Asai: Sens. Mater. Mater. 31 (2019) 1241.

21 M. Sekita, Y. Miyazawa, T. Akahane, and T. Chiba: J. Appl. Phys. 66 (1989) 373.

22 H. Suzuki, T. A. Tombrello, C. L. Melcher, and J. S. Schweitzer: Nucl. Instrum. Methods Phys. Res., Sect. A 320 (1992) 263.

23 H. Suzuki, T. A. Tombrello, C. L. Melcher, and J. S. Schweitzer: IEEE Trans. Nucl. Sci. 40 (1993) 380.

24 H. Suzuki, T. A. Tombrello, C. L. Melcher, and J. S. Schweitzer: IEEE Trans. Nucl. Sci. 41 (1994) 681.

25 H. Suzuki, T. A. Tombrello, C. L. Melcher, C. A. Peterson, and J. S. Schweitzer: Nucl. Instrum. Methods Phys. Res., Sect. A 346 (1994) 510.

26 K. Mori, M. Yokoya, H. Nishimura, and M. Nakayama: Int. J. Mod. Phys. B 15 (2001) 3877.

27 C. L. Melcher, R. A. Manente, C. A. Peterson, and J. S. Schweitzer: J. Cryst. Growth 128 (1993) 1001.

28 H. Suzuki, T. A. Tombrello, C. L. Melcher, and J. S. Schweitzer: IEEE Trans. Nucl. Sci. 40 (1993) 380. 